\title{
THE ASSOCIATION OF WHITE MATTER INTEGRITY AND AGE ON COGNITIVE PERFORMANCE IN U-2 PILOTS
}

Paul Sherman MD ${ }^{1,2}$, Bianca Cerqueira ${ }^{1,3}, \mathrm{PhD}$, John Sladky, MD ${ }^{1,2}$. USAF School of Aerospace Medicine; Dept of Radiology and Neurology, 59 MDW; KBR Aerospace

\section{Introduction}

Repeated occupational exposure to hypobaric conditions results in reduced white matter integrity as shown by increased white matter hyper-intensity (WMH) burden and decreased fractional anisotropy (FA) on MRI.

\section{Background}

WMH are a marker of cerebral integrity and associated with cognitive decline. Both U-2 pilots and aerospace physiological personnel display an increased incidence of $\mathrm{WMH}$ on MRI compared to healthy age-matched controls (non-pilots). U-2 pilots demonstrate an apparent acquired decrement in axonal integrity based on FA measurements. U-2 pilots demonstrate reduced neurocognitive performance associated with burden of $\mathrm{WMH}$. Increase in WMH volume and decline in FA values are also observed in normal WM aging.

\section{Methods}

103 U-2 pilots (ages 28-58), 83 physiology/chamber technicians (ages 28-58), and 162 controls (ages 28-58).

MRI included 3D FLAIR and diffusion tensor imaging sequences

to acquire $F A$ values/assess white matter integrity.

$\mathrm{U}-2$ pilots underwent MAB and microcognitive (MicroCog) assessment.

Used generalized linear model (GLM) for effects of age, group, and interaction

\section{Results}

WMH Volume: Group Comparisons

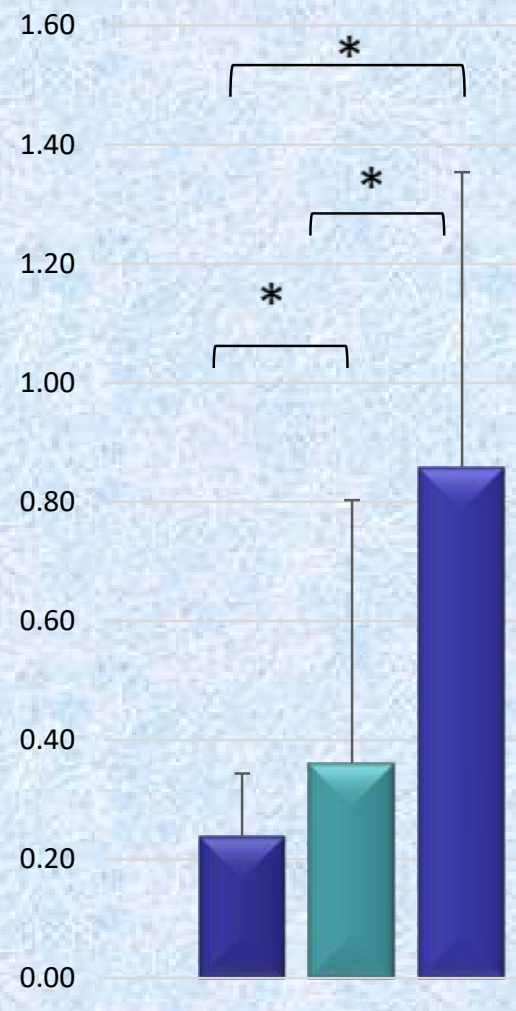

total

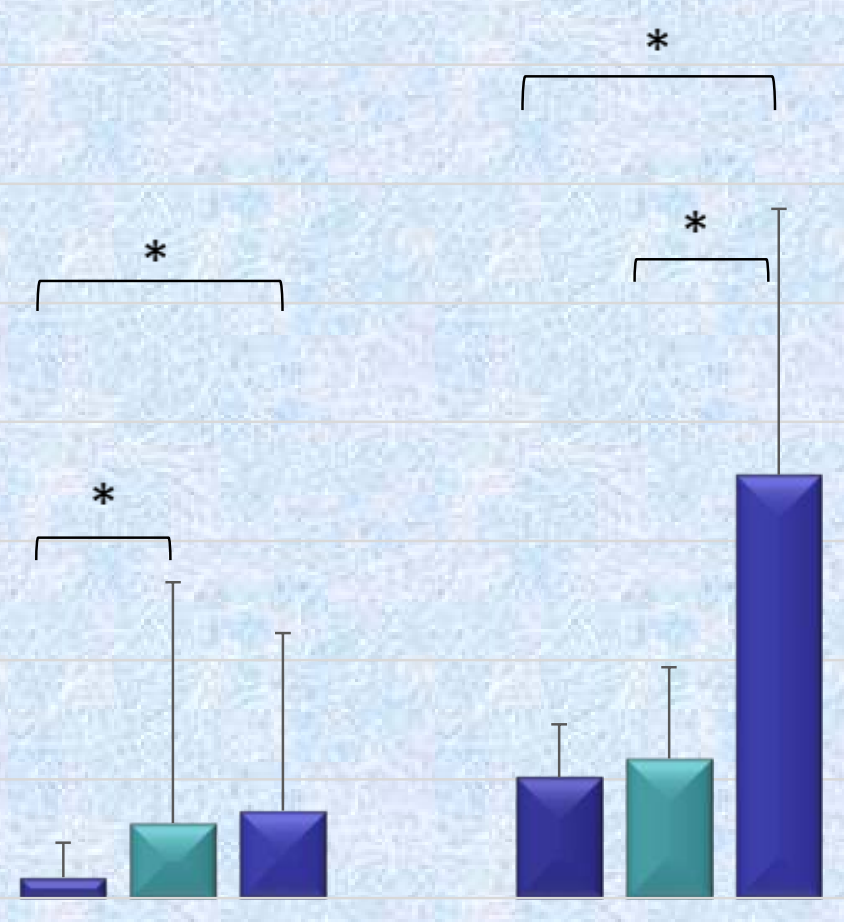

subcortical

periependymal

Total FLAIR WMH Volume Over Time

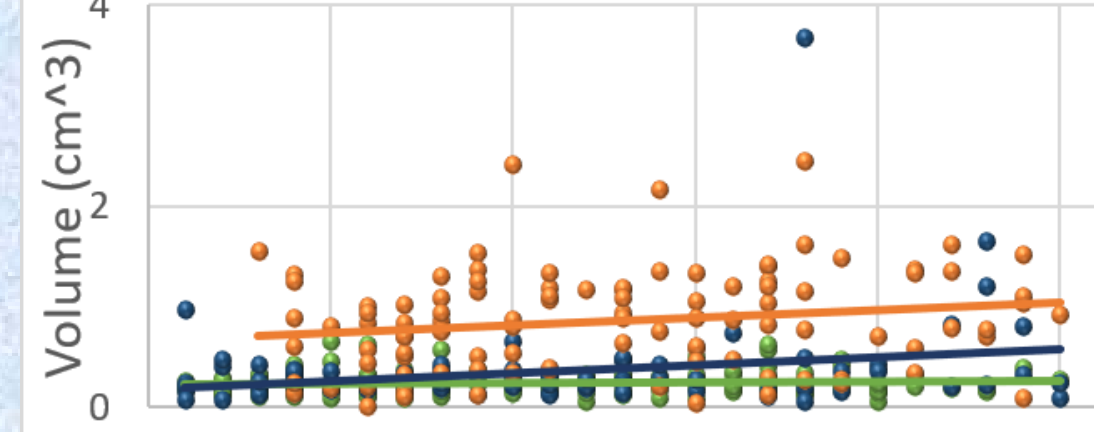

- DOC Age $\quad$ PHY

MicroCog scores lower in U2P with higher WMH burden

\begin{tabular}{|c|c|c|c|c|c|c|c|c|}
\hline \multirow[b]{2}{*}{ MicroCog } & \multicolumn{2}{|c|}{$\begin{array}{l}\text { Lower WMH (mean count/volume } \\
0.6 / 0.003 \mathrm{~mL} \text { ) }\end{array}$} & \multicolumn{2}{|c|}{$\begin{array}{l}\text { Upper WMH (mean count/volume } \\
14.4 / 0.213 \mathrm{~mL} \text { ) }\end{array}$} & \multicolumn{2}{|c|}{$\begin{array}{l}\text { p Value, } t \text { test } \\
\text { (2-tailed) }\end{array}$} & \multicolumn{2}{|c|}{$\begin{array}{l}\text { p Value, Sidak } \\
\text { (2-tailed) }\end{array}$} \\
\hline & Count $(n=33)$ & Volume $(n=30)$ & Count $(n=60)$ & Volume $(n=63)$ & Count & Volume & Count & Volume \\
\hline Attention/mental control & $104.8 \pm 6.7$ & $104.7 \pm 7.2$ & $104.2 \pm 10.4$ & $104.2 \pm 10.1$ & 0.808 & 0.806 & 1.000 & 1.000 \\
\hline Reasoning/calculation & $104.1 \pm 11.3$ & $101.8 \pm 11.7$ & $96.8 \pm 12.3$ & $98.2 \pm 12.6$ & 0.009 & 0.197 & 0.044 & 0.666 \\
\hline Memory & $110.2 \pm 11.0$ & $108.8 \pm 12.5$ & $102.9 \pm 12.4$ & $103.9 \pm 12.1$ & 0.006 & 0.075 & 0.030 & 0.323 \\
\hline Spatial processing & $111.0 \pm 8.3$ & $110.9 \pm 8.3$ & $108.1 \pm 9.8$ & $108.3 \pm 9.7$ & 0.161 & 0.202 & 0.584 & 0.676 \\
\hline Reaction time & $108.4 \pm 6.1$ & $109.5 \pm 5.4$ & $106.7 \pm 6.9$ & $106.2 \pm 7.0$ & 0.299 & 0.028 & 0.831 & 0.132 \\
\hline IPS & $106.7 \pm 11.5$ & $1047 \pm 13.3$ & $101.9 \pm 12.5$ & $103.0 \pm 11.9$ & 0.101 & 0.534 & 0.192 & 0.783 \\
\hline PA & $105.0 \pm 7.9$ & $105.2 \pm 8.6$ & $100.5 \pm 10.2$ & $100.7 \pm 9.9$ & 0.029 & 0.036 & 0.057 & 0.071 \\
\hline GCF & $107.2 \pm 8.9$ & $106.1 \pm 9.8$ & $101.5 \pm 9.8$ & $102.3 \pm 9.7$ & 0.010 & 0.081 & 0.020 & 0.155 \\
\hline GCP & $108.8 \pm 8.6$ & $107.6 \pm 9.0$ & $103.6 \pm 9.3$ & $104.4 \pm 9.4$ & 0.011 & 0.121 & 0.022 & 0.227 \\
\hline
\end{tabular}
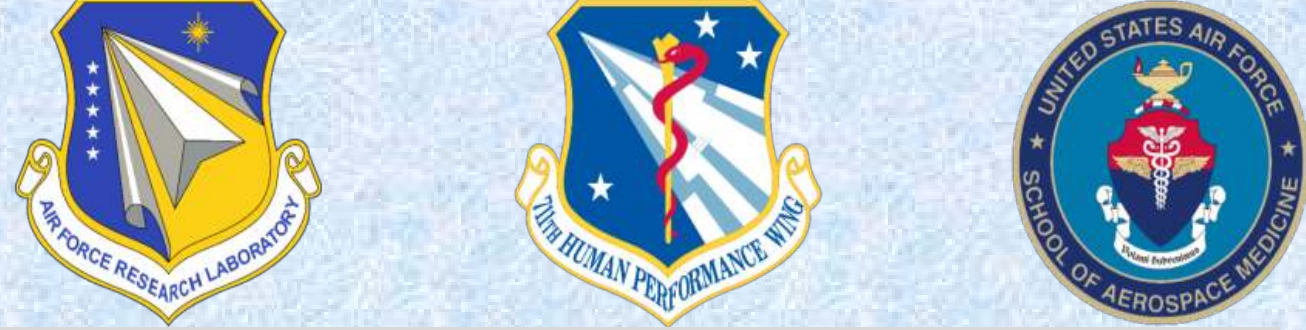

Average FA Over Time

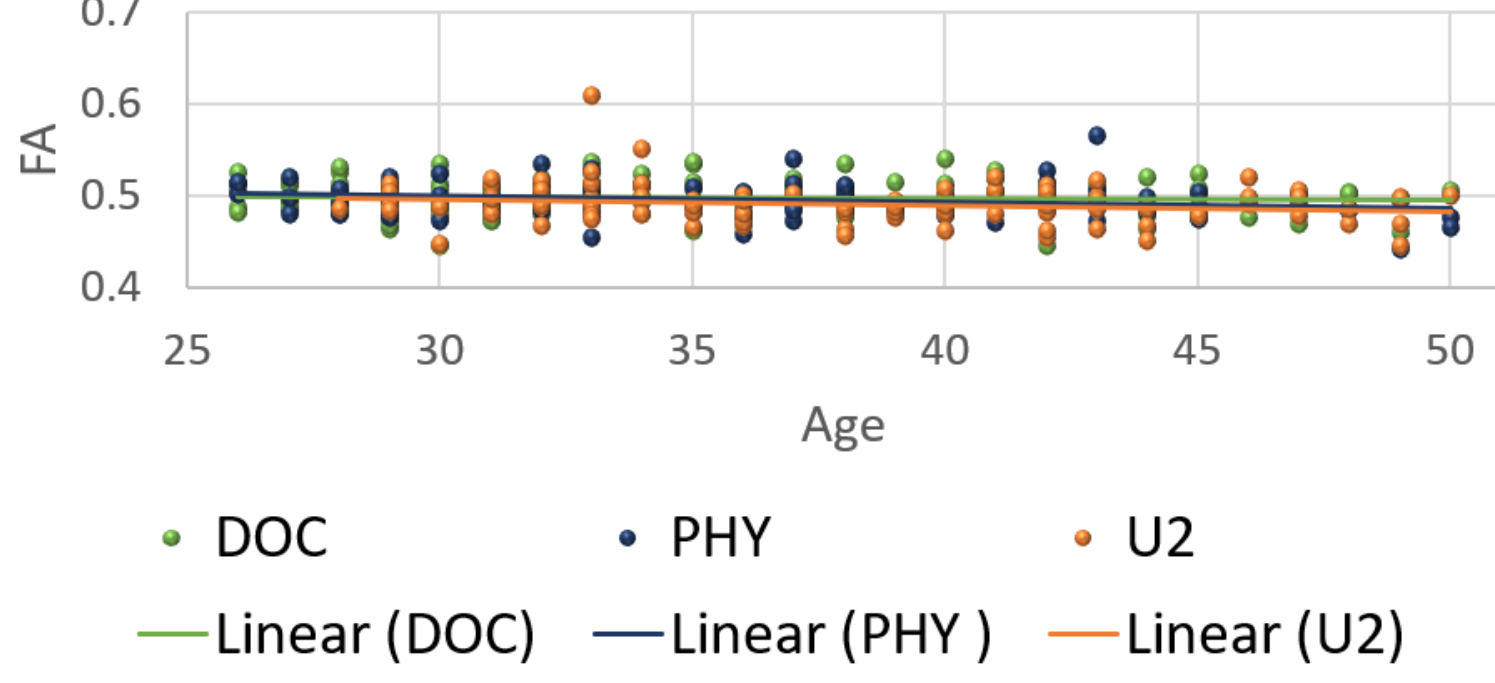

Effect of FA on MircoCog Scores

\begin{tabular}{|c|c|c|c|c|c|c|c|c|}
\hline & & $\begin{array}{c}\text { Corpus } \\
\text { Callosum } \\
\text { Body }\end{array}$ & Fornix & $\begin{array}{c}\text { Internal } \\
\text { Capsule }\end{array}$ & $\begin{array}{c}\text { Corona } \\
\text { Radiata }\end{array}$ & $\begin{array}{c}\text { External } \\
\text { Capsule }\end{array}$ & $\begin{array}{c}\text { Fronto- } \\
\text { occipital }\end{array}$ & $\begin{array}{c}\text { Superior } \\
\text { Fronto- } \\
\text { occipital }\end{array}$ \\
\hline $\mathbf{1}$ & Attention & 0.8555 & 0.3077 & 0.3923 & 0.7063 & 0.251 & 0.8146 & 0.7185 \\
\hline $\mathbf{1}$ & Reason & 0.6974 & 0.4162 & 0.8526 & 0.8742 & 0.9397 & 0.3359 & 0.2474 \\
\hline $\mathbf{1}$ & Memory & 0.6974 & 0.9523 & 0.3801 & 0.2496 & 0.7029 & 0.9499 & 0.9999 \\
\hline $\mathbf{1}$ & $\begin{array}{c}\text { Spatial } \\
\text { Processing }\end{array}$ & $* 0.0058$ & 0.2704 & $* 0.0141$ & $* 0.0062$ & $* 0.0059$ & $* 0.0359$ & 0.4049 \\
\hline $\mathbf{1}$ & $\begin{array}{c}\text { Reaction } \\
\text { Time }\end{array}$ & 0.3823 & 0.1436 & 0.1493 & 0.2483 & 0.3257 & $\neq 0.0335$ & 0.283 \\
\hline $\mathbf{2}$ & Speed & 0.9962 & 0.9834 & 0.1075 & 0.2437 & 0.5248 & 0.6299 & 0.6088 \\
\hline $\mathbf{2}$ & Accuracy & 0.334 & 0.3044 & 0.6897 & 0.4949 & 0.1623 & 0.7929 & 0.4689 \\
\hline $\mathbf{3}$ & Function & 0.5804 & 0.55 & 0.1256 & 0.1928 & 0.1698 & 0.824 & 0.9307 \\
\hline $\mathbf{3}$ & Proficiency & 0.8985 & 0.6976 & 0.2462 & 0.3928 & 0.3653 & 0.967 & 0.6114 \\
\hline
\end{tabular}

\section{GLM with FA and Age as Independent Variables}

\begin{tabular}{|c|c|c|c|c|c|c|c|c|}
\hline & $\begin{array}{l}\text { Corpus } \\
\text { Callosum } \\
\text { Body }\end{array}$ & Age & & Fornix & $\begin{array}{c}\mathrm{Ag} \\
\mathrm{e}\end{array}$ & & $\begin{array}{l}\text { Internal } \\
\text { Capsule }\end{array}$ & Age \\
\hline Attention & ns & ns & Attention & ns & $\begin{array}{l}0.0 \\
31^{*}\end{array}$ & Attention & ns & $\begin{array}{c}0.04 \\
5^{*}\end{array}$ \\
\hline Reason & ns & ns & Reason & ns & ns & Reason & ns & ns \\
\hline & ns & ns & & ns & $\mathrm{ns}$ & Men & ns & ns \\
\hline $\begin{array}{l}\text { Spatial } \\
\text { Processing }\end{array}$ & $0.019^{*}$ & ns & $\begin{array}{c}\text { Spatial } \\
\text { Processing }\end{array}$ & ns & ns & $\begin{array}{c}\text { Spatial } \\
\text { Processing }\end{array}$ & $0.023^{*}$ & ns \\
\hline $\begin{array}{c}\text { Reaction } \\
\text { Time }\end{array}$ & ns & $\begin{array}{c}0.03 \\
6^{*}\end{array}$ & $\begin{array}{c}\text { Reaction } \\
\text { Time }\end{array}$ & ns & $\begin{array}{l}0.0 \\
22 *\end{array}$ & $\begin{array}{c}\text { Reaction } \\
\text { Time }\end{array}$ & ns & $\begin{array}{c}0.01 \\
7^{*}\end{array}$ \\
\hline Speed & ns & ns & Speed & ns & ns & Spe & ns & ns \\
\hline Acc & ns & ns & Acc & ns & ns & Acc & ns & רs \\
\hline & ns & ns & Fur & $n s$ & ns & Fu & $\mathrm{ns}$ & nis \\
\hline Proficiency & ns & ns & Profici & ns & ns & Profi & ns & ns \\
\hline & $\begin{array}{l}\text { External } \\
\text { Capsule }\end{array}$ & Age & & $\begin{array}{l}\text { Fronto } \\
- \\
\text { occipit } \\
\text { al }\end{array}$ & $\mathrm{Ag}$ & & $\begin{array}{l}\text { Superior } \\
\text { Fronto- } \\
\text { occipital }\end{array}$ & $A g$ \\
\hline Attention & ns & $\begin{array}{c}0.03 \\
1^{*}\end{array}$ & Attention & ns & ns & Attention & ns & $\mathrm{ns}$ \\
\hline on & ns & $\begin{array}{c}0.04 \\
96^{*}\end{array}$ & $\mathbf{R}$ & ns & ns & eason & ns & ns \\
\hline Memory & ns & ns & Memory & ns & ns & Memory & ns & ns \\
\hline $\begin{array}{c}\text { Spatial } \\
\text { Processing }\end{array}$ & $0.015^{*}$ & ns & $\begin{array}{c}\text { Spatial } \\
\text { Processing }\end{array}$ & $0.046^{*}$ & $\begin{array}{c}0.0 \\
44 \\
\neq\end{array}$ & $\begin{array}{c}\text { Spatial } \\
\text { Processing }\end{array}$ & ns & 0.6 \\
\hline $\begin{array}{c}\text { Reaction } \\
\text { Time }\end{array}$ & ns & $\begin{array}{c}0.01 \\
7^{*}\end{array}$ & $\begin{array}{c}\text { Reaction } \\
\text { Time }\end{array}$ & $0.045 \neq$ & $\begin{array}{l}0.0 \\
15^{*}\end{array}$ & $\begin{array}{c}\text { Reaction } \\
\text { Time }\end{array}$ & ns & 0.0 \\
\hline Speed & ns & ns & Speed & ns & ns! & & $\mathrm{n}$ & \\
\hline Acc & ns & ns & acy & ns & $\mathrm{ns}$ & Acc & ns & \\
\hline & ns & ns & Fur & ns & ns & & ns & \\
\hline Proficiency & ns & $\mathrm{ns}$ & Proficiency & ns & $\mathrm{ns}$ & Proficiency & ns & \\
\hline
\end{tabular}

\section{Discussion - Conclusions}

In U2P, both age and FA values independently influence certain microcognitive tracts

Positive correlation to spatial processing when controlling for age Increasing age correlates with improved reaction time independent of FA values for all tracts

Suggests role of repetitive memory with increased age (long-term potential)

Occupational exposure to hypobaria does not result in acceleration of cerebral aging

\section{Bibliography}

Kochunov $P$, Robin DA, Royall DR, et al. Can structural MRI indices of cerebral integrity track cognitive trends in executive control function during normal maturation and adulthood? Hum Brain Mapp. 2009; 30(8):25812594 Kochunov P. Thompson PM, Coyle TR, et al. Relationship among neuroimaging indices of cerebral health during normal aging. Hum Brain Mapp. 2008; 29(1):36-45. McGuire S, Sherman P, Profenna L, et al. White matter hyperintensities on MRI in high-altitude U-2 pilots. Neurology. 2013; 81(8):729-735. McGuire SA, Sherman PM, Wijtenburg SA, et al. White matter hyperintensities and hypobaric exposure. Ann Neurol. 2014; 76(5):719-726. McGuire SA, Tate DF, Wood J, et al. Lower neurocognitive function in high-altitude U-2 pilots: Relationship to white matter hyperintensities. Neurology. 2014; 83(7):638-645. 